\title{
Comparação das variáveis eletromiográficas e cinemáticas entre uma corrida do "triathlon" e uma corrida prolongada
}

CDD. 20.ed. 796.023

796.426

\author{
Carina Helena Wasem FRAGA* \\ Roberto BIANCO* \\ Júlio Cerca SERRÃO* \\ Pedro Ernesto Sales de SOUZA** \\ Alberto Carlos AMADIO* \\ Antônio Carlos Stringhini GUIMARÃES (in memorian) ${ }^{* * *}$ \\ Marco Aurélio VAZ ${ }^{* * *}$
}

\section{Resumo}

A corrida e o ciclismo realizados com uma mesma duração e intensidade podem apresentar diferentes respostas biomecânicas e metabólicas durante um protocolo de fadiga devido à sobrecarga mecânica e à especificidade técnica de cada modalidade. 0 objetivo do presente estudo foi avaliar os efeitos da fadiga proveniente de uma corrida de $10 \mathrm{~km}$, precedida por ciclismo ou corrida, no padrão de passada e no sinal eletromiográfico (EMG). Nove triatletas do sexo masculino com tempo de prática superior a dois anos participaram do estudo. Os testes foram realizados em duas etapas: corrida do "triathlon" (40 km de ciclismo seguidos de $10 \mathrm{~km}$ de corrida) e corrida prolongada (corrida com duração igual ao tempo que o atleta levou para percorrer os $40 \mathrm{~km}$ de ciclismo, seguidos de mais $10 \mathrm{~km}$ de corrida). Uma análise cinemática (frequência e amplitude de passada) e eletromiográfica correspondentes às 10 passadas registradas no $5^{\circ} \mathrm{km}$ de cada corrida foi realizada. As curvas de EMG foram retificadas e filtradas para cálculo das curvas de RMS. A partir da média das curvas de EMG foram obtidos os valores de pico de RMS para os músculos reto femoral, vasto lateral e bíceps femoral. Maiores valores de frequência de passada e do sinal EMG do músculo bíceps femoral foram obtidos na corrida prolongada quando comparada com a corrida do "triathlon". Esses resultados parecem estar relacionados a maior fadiga proveniente da corrida prolongada devido às maiores exigências mecânicas.

Palavras-Chave: Corrida; "Triathlon"; Fadiga; Eletromiografia; Cinemática.

\section{Introdução}

Diferentes exercícios de mesma duração, realizados na mesma intensidade, podem apresentar diferentes respostas metabólicas e biomecânicas, devido à especificidade técnica de cada modalidade ${ }^{1}$. Dessa forma, há inúmeras adaptações específicas, inerentes à prática da corrida e do ciclismo, que proporcionam respostas tanto fisiológicas ${ }^{2}$ como biomecânicas ${ }^{3}$ diferenciadas.

O "triathlon", composto por três modalidades realizadas sucessivamente (natação, ciclismo e corrida), apresenta especificidades que desencadeiam demandas fisiológicas e biomecânicas diferentes dos esportes individuais que o compõem ${ }^{1}$.

Neste esporte, a corrida representa um importante segmento, tendo em vista que seu desempenho pode afetar significativamente o resultado do restante da prova ${ }^{2,4}$.

Pela especificidade do movimento de cada modalidade que compõe o "triathlon", o recrutamento de unidades motoras pode ser diferente entre o ciclismo e a corrida, o que pode resultar em diferentes reservas de glicogênio disponíveis no início de uma corrida subsequentes. Assim, presume-se que os níveis de fadiga induzidos pelo "triathlon" sejam menores do que uma prova com mesma duração que envolva apenas uma modalidade esportiva (como por exemplo, uma corrida prolongada).

Em corridas prolongadas como a maratona, HAUSSWIRTH et al. ${ }^{5}$ verificaram redução na economia da corrida ao longo da prova associada a uma diminuição 
na frequência da passada. Dessa forma, a relação entre os parâmetros cinemáticos e de economia do movimento salientam a importância da seleção adequada de variáveis biomecânicas, como a amplitude e a frequência da passada, para melhora do desempenho da corrida.

Além disso, o desempenho em exercícios prolongados é limitado pela capacidade do sistema aeróbico em fornecer um suprimento de energia de forma contínua, o qual é necessário para contração muscular ${ }^{6}$. Uma deficiência no suprimento de energia tem sido associada com uma redução do desempenho devido à fadiga muscular ${ }^{7}$.

A eletromiografia de superfície tem sido utilizada para comparar a atividade muscular entre diferentes movimentos no esporte, sendo uma técnica que possibilita a avaliação da ativação muscular, coordenação motora e fadiga. Nesse sentido, a fadiga representa um indicador de diminuição da força e potência ${ }^{8}$, que pode ocorrer durante a execução de exercícios prolongados e/ou de alta intensidade.

Sabe-se que tanto a corrida prolongada como aquela realizada em alta intensidade pode induzir a modificações nos parâmetros eletromiográficos e cinemáticos decorrentes de um processo de fadiga ${ }^{9-10}$.

\section{Método}

Participaram deste estudo nove triatletas do sexo masculino (TABELA 1), registrados na Federação Gaúcha de Triathlon, os quais possuíam um tempo de prática na modalidade superior a dois anos.

Todos os atletas assinaram um Termo de Consentimento Livre e Esclarecido concordando com sua participação voluntária neste estudo. $\mathrm{O}$ projeto foi submetido e aprovado pelo Comitê de Pesquisa Local.

\section{Protocolo de avaliação}

Os testes foram realizados em duas etapas: 1) corrida do "triathlon": $40 \mathrm{~km}$ de ciclismo seguidos de $10 \mathrm{~km}$ de corrida - distâncias oficiais das provas do Triathlon Olímpico; 2) corrida prolongada: corrida com duração igual ao tempo que o atleta levou para percorrer os $40 \mathrm{~km}$ de ciclismo, seguidos de mais $10 \mathrm{~km}$ de corrida. Os testes foram realizados com um intervalo mínimo de 48 horas entre eles, na tentativa de evitar que a fadiga pudesse interferir nos resultados entre os testes. A viabilidade desse protocolo foi testada previamente por meio de um estudo piloto ${ }^{15}$.
Dessa forma, o conhecimento sobre o padrão de ativação dos músculos do membro inferior é importante para o entendimento de aspectos cinemáticos da corrida ${ }^{11}$. Esse entendimento é essencial, tendo em vista que, embora a corrida possa ser classificada como uma atividade de deslocamento natural do ser humano, fatores como a escolha adequada da amplitude e frequência de passada pode influenciar o desempenho durante a corrida ${ }^{12-14}$.

Diante do exposto, esse estudo tem por objetivo analisar a fadiga proveniente de exercícios prolongados, e determinar se diferentes atividades que precedem uma corrida de $10 \mathrm{~km}$ (ciclismo ou corrida) podem acarretar alteraçōes no padrão de passada e no sinal EMG. A hipótese deste estudo é que a corrida prolongada, por tratar-se de uma mesma atividade realizada por um longo período de tempo e que envolve a sustentação do peso corporal, induzirá a maior fadiga. Com isso, por meio da comparação entre a corrida do "triathlon" e a corrida prolongada, tem-se como hipótese que serão verificados maiores valores do sinal EMG, maiores valores de frequência de passada e menores valores de amplitude de passada na corrida prolongada.

Uma análise cinemática e eletromiográfica foi realizada em 10 passadas registradas no $5^{\circ} \mathrm{km}$ da corrida do "triathlon" e da corrida prolongada. O tempo de transição ciclismo-corrida e corrida-corrida foi de aproximadamente um minuto, e foi semelhante ao tempo de transição de uma prova de "triathlon".

No intuito de simular a intensidade real de prova, foi realizada uma competição entre os atletas, do tipo "contra-relógio" na corrida do" triathlon", para a qual foi estabelecida premiação previamente divulgada. A velocidade média obtida nessa corrida foi posteriormente utilizada para padronizar a corrida prolongada - sendo que essa velocidade foi calculada a partir da média da velocidade registrada em cada um dos $10 \mathrm{~km}$ da corrida do "triathlon".

Para o teste que envolveu o ciclismo, foi utilizado um ciclo simulador da marca Cateye, modelo CS1000 (Cateye, Japão), o qual permitiu que a avaliação fosse realizada na própria bicicleta do atleta.

Os atletas utilizaram os ajustes habituais em suas bicicletas de acordo com a escolha individual. Não foi utilizada qualquer padronização para que se criasse uma situação mais aproximada da condição 
de prova. A cadência de pedalada também foi determinada pela livre escolha do atleta.

Para avaliação da corrida foi utilizada uma esteira motorizada (marca Quinton, USA).

\section{Aquisição de dados cinemáticos}

Um sistema de cinemetria Peak Motus (Peak Performance, Inc., USA) foi utilizado para uma análise bidimensional no plano sagital de movimento (cinemetria 2D). Um módulo de sincronismo permitiu que os sinais eletromiográficos fossem coletados simultaneamente aos dados cinemáticos. Uma câmera (marca Pulmix, UK; frequência de amostragem de $60 \mathrm{~Hz}$ ) foi posicionada a uma distância de 4,25 m da esteira, em uma altura de $90 \mathrm{~cm}$.

Para definição da escala linear uma régua de calibração de um metro de comprimento foi posicionada no local de captação das imagens, no início da filmagem. Um marcador reflexivo, com aproximadamente $2 \mathrm{~cm}^{2}$, foi posicionado no ponto anatômico de referência do calcanhar esquerdo (região do calcâneo esquerdo).

\section{Aquisição de eletromiográficos}

Para o registro do sinal EMG foram utilizados eletrodos passivos de superfície $(\mathrm{Ag} / \mathrm{AgCl})$ na configuração bipolar e adesivo de fixação com diâmetro de $2,2 \mathrm{~cm}$. Os eletrodos foram alinhados longitudinalmente às fibras musculares e fixados sobre a pele que recobria o ventre dos músculos bíceps femoral, reto femoral e vasto lateral, do lado esquerdo do corpo, com posicionamento seguindo as normas de acordo com SENIAM (Surface Electromyography for the Non-Invasive Assessment of Muscles) ${ }^{16}$. O eletrodo de referência foi fixado sobre a pele recobrindo a face anterior da tíbia.

Os sinais EMG foram obtidos de cada um dos músculos com uma frequência de $2000 \mathrm{~Hz}$. Todas as normas pertinentes ao registro adequado de sinais EMG foram rigorosamente observadas, como tricotomia, limpeza do local com álcool, colocação dos eletrodos, sugeridas por MERLETTI ${ }^{17}$ e recomendados pela Sociedade Internacional de Eletrofisiologia e Cinesiologia ${ }^{18}$.

Para a obtenção do sinal EMG, foi utilizado um conversor análogo-digital de 16 canais CODAS (Dataq Instruments, Inc., Akron, USA) e um eletromiógrafo de oito canais com amplificação de $1 \mathrm{~K}$ (Bortec Eletronics Inc., Calgary, Canada).
A coleta do sinal eletromiográfico foi realizada simultaneamente com os dados cinemáticos, utilizando um módulo de sincronismo. O momento inicial de análise dos dados cinemáticos e eletromiográficos foi considerado o primeiro toque do calcanhar no solo, após o pulso de sincronismo.

\section{Análise das variáveis cinemáticas}

Dez passadas foram analisadas a partir do $5^{\circ} \mathrm{km}$ de cada corrida (corrida do "triathlon" e corrida prolongada). Para a digitalização automática das imagens foi utilizado o sistema Peak Motus (Peak Performance, Inc., USA), o qual possibilitou o cálculo de coordenadas espaciais do ponto do calcâneo em relação a um referencial inercial. Após a imagem ser adquirida e digitalizada, os dados foram expostos a um filtro do tipo Butterwoth de ordem quatro, adotando-se uma frequência de corte de $6 \mathrm{~Hz}$.

As 10 passadas analisadas foram divididas por sucessivos toques do calcâneo com o solo (obtidos por meio dos dados referentes às coordenadas espaciais), o que permitiu a normalização considerando $100 \%$ de um ciclo médio de passada.

As coordenadas referentes às distâncias percorridas pelo ponto do calcâneo no eixo horizontal de movimento permitiram o cálculo da amplitude de passada (expressa em metros), enquanto que os parâmetros temporais associados ao momento do toque do calcâneo no solo foram utilizados para o cálculo da frequência de passada (expressa em número de passadas por minuto).

\section{Análise das variáveis eletromiográficas}

Para o processamento dos sinais EMG foi utilizado o sistema SAD32 de aquisição de dados (versão 2.61.07mp, 2002). O sinal EMG foi exposto a um filtro digital passa-banda do tipo Butterworth de ordem cinco, com frequência de corte de 10 a 500 $\mathrm{Hz}$, e, posteriormente, analisado no domínio do tempo, a partir do cálculo de um envoltório linear, em intervalos de $40 \mathrm{~ms}$ (janelamento de Hamming) ${ }^{19}$. Não foi utilizado nenhum filtro para ruídos de rede, pois a alimentação do sistema ocorreu com uso de uma bateria independente, e não pela rede elétrica. Uma curva média foi obtida a partir do registro dos 10 ciclos de passada, sendo esta normalizada no eixo vertical pelos valores de pico ${ }^{20,21}$, e no eixo horizontal considerando $100 \%$ do ciclo de passada. O valor de referência utilizado para normalização no eixo vertical foi o pico obtido em 10 ciclos de passada no 
$1^{\circ} \mathrm{km}$ da corrida prolongada, considerando que nessa situação o sinal sofreu as menores influências da fadiga, decorrentes de atividades prolongadas. Os valores máximos obtidos em cada uma das curvas médias foram analisados em cada corrida para cada músculo.

\section{Análise estatística dos dados}

Inicialmente foram calculados as médias e os desvios padrão para todas as variáveis. A seguir, foi

\section{Resultados}

Na TABELA 1 são apresentadas algumas das características da amostra desse estudo.

Na FIGURA 1 são apresentados os resultados referentes à velocidade de teste. $\mathrm{Na}$ corrida do "triathlon" a velocidade foi determinada pelo próprio atleta, sendo que a média do grupo avaliado avaliada a normalidade da distribuição dos dados com teste de Shapiro-Wilk.

Para comparação das variáveis cinemáticas e eletromiográficas entre o $5^{\circ} \mathrm{km}$ de corrida do "triathlon" e $5^{\circ} \mathrm{km}$ da corrida prolongada, foi aplicado um teste $t$ de Student para dados pareados.

Um nível de significância de $p<0,05$ foi considerado para todas as análises. O tratamento estatístico foi realizado com o aplicativo SPSS (Statistical Package for Social Sciences), versão 13.0.

TABELA 1 - Características da amostra.

reflete uma estratégia incremental ao longo de todo teste $\left(\mathrm{de} 13,4 \mathrm{~km} \cdot \mathrm{h}^{-1}\right.$ no $1^{\circ} \mathrm{km}$ para $14,4 \mathrm{~km} \cdot \mathrm{h}^{-1}$ no $9^{\circ} \mathrm{km}$ ). A velocidade da corrida prolongada foi mantida constante, sendo correspondente à média da velocidade registrada em cada um dos $10 \mathrm{~km} \mathrm{da}$ corrida do "triathlon" (14 km.h $\left.\mathrm{h}^{-1}\right)$.

\begin{tabular}{lcccc}
\hline & Idade (anos) & Estatura $(\mathbf{m})$ & Massa $(\mathbf{k g})$ & Tempo de treino (anos) \\
\hline Médias e Desvios-padrão & $32 \pm 5,87$ & $1,7 \pm 0,05$ & $76,43 \pm 6,61$ & $5,33 \pm 3,28$ \\
\hline
\end{tabular}

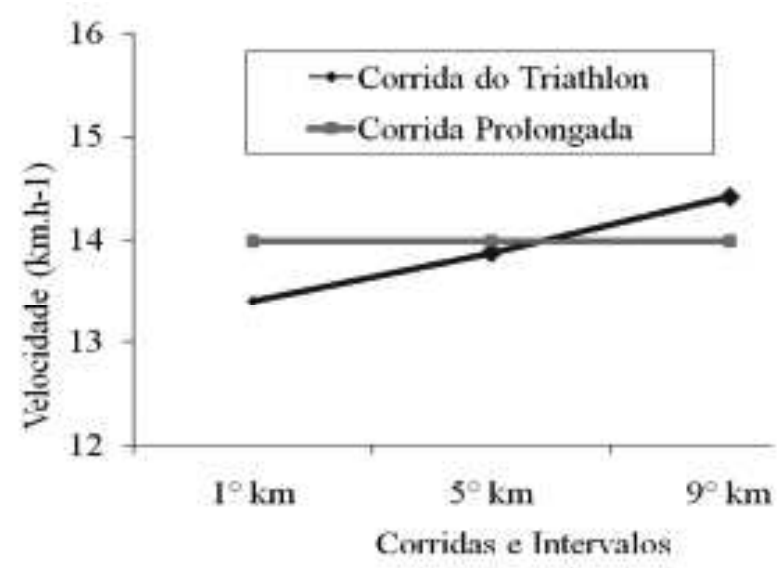

FIGURA 1 - Velocidade média da corrida do "triathlon" e da corrida prolongada no $1^{\circ}, 5^{\circ}$ e $9^{\circ} \mathrm{km}$.

Na TABELA 2 são apresentados os valores de média e desvio-padrão das variáveis cinemáticas analisadas.

A frequência de passada foi significativamente maior na corrida prolongada $(\mathrm{p}=0,014)$, enquanto a amplitude de passada não apresentou diferença entre as corridas. Entretanto, considerando que a velocidade representa o produto direto entre essas duas variáveis e que a velocidade média da corrida prolongada foi a mesma da corrida do "triathlon", já se esperava uma redução da amplitude de passada na corrida prolongada a partir do aumento da frequência de passada, mesmo que essa diminuição não fosse significativa.

Quando comparado o sinal EMG entre as duas corridas analisadas, foram verificados maiores valores apenas para o músculo bíceps femoral na corrida prolongada $(\mathrm{p}=0,005)$, (FIGURA 2). Os demais músculos não apresentaram diferenças significativas entre as duas corridas analisadas. 
TABELA 2 - Valores correspondentes a média e o desvio padrão da amplitude e frequência de passada.

\begin{tabular}{lcc}
\hline & Corrida do "Triathlon" & Corrida prolongada \\
\hline Amplitude da passada $(\mathrm{m})$ & $2,10 \pm 0,38$ & $1,98 \pm 0,30$ \\
Frequência de passada (passada/min) & $85,72 \pm 3,27^{*}$ & $87,97 \pm 3,32$ \\
\hline
\end{tabular}

${ }^{*} p<0,05$ comparado à corrida prolongada.

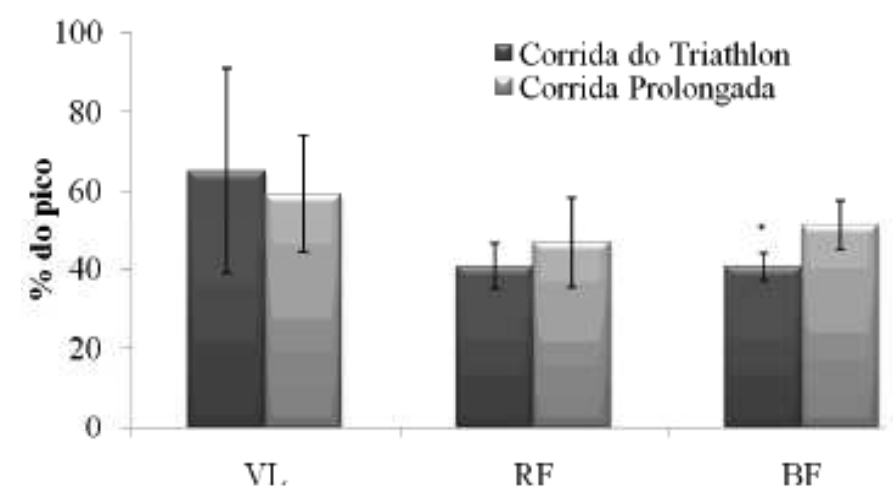

FIGURA 2 - Valor máximo obtido na curva média das 10 passadas do $5^{\circ} \mathrm{km}$ da corrida do "triathlon" e da corrida prolongada, para os músculos vasto lateral (VL), reto femoral (RF) e bíceps femoral (BF).

\section{Discussão}

O objetivo do presente estudo foi o de determinar os efeitos de diferentes atividades (ciclismo ou corrida) sobre o padrão de passada e o sinal EMG em uma corrida de $10 \mathrm{~km}$. A hipótese deste estudo era de que a corrida prolongada resultaria em maiores valores do sinal EMG pelo desgaste advindo de uma mesma atividade realizada por um período de tempo prolongado (a qual, diferentemente do ciclismo, envolve sustentação do peso corporal). Além disso, esperava-se que fossem encontrados maiores valores de frequência de passada, e menores valores de amplitude de passada na corrida prolongada, uma vez que a velocidade média de corrida foi a mesma. Essa última hipótese está associada ao fato de que um aumento da frequência de passada está relacionado a um maior gasto de energia, e, portanto, maior suscetibilidade a processos de fadiga induzidos pela corrida prolongada.

O maior gasto energético, durante a corrida prolongada, pode ocorrer devido à necessidade de um maior número de ciclos de passada que exige concomitantes alterações nos níveis de alongamento-encurtamento muscular, bem como na taxa de força desenvolvida, o que afeta diretamente a demanda aeróbica ${ }^{22}$.

LEPERS et al. ${ }^{23}$ verificaram que não há ocorrência de fadiga adicional com o uso de diferentes cadências durante o ciclismo. Desta forma, a escolha dos atletas pela cadência que estão mais habituados não deve ter influenciado a corrida subsequente, devido sua familiaridade com a mesma ${ }^{4}$.

Para as variáveis cinemáticas analisadas foi verificado aumento da frequência de passada na corrida prolongada comparada à corrida do "triathlon". Entretanto, não foram verificadas diferenças significativas na amplitude de passada. $\mathrm{TEW}^{24}$ verificou que o aumento da velocidade de corrida esteve relacionado com um aumento da frequência de passada, sem alterações na amplitude de passada, o que corrobora, em parte, com os resultados verificados no presente estudo. Essas alterações na velocidade de corrida ou na frequência de passada podem influenciar outros parâmetros do movimento, e resultar na variabilidade de seu desempenho ${ }^{13,25-26}$.

Em relação à estratégia utilizada pelos triatletas, CAVANAGH e Kram ${ }^{27}$ e Brisswalter et al. ${ }^{28}$ afirmam que em uma corrida de velocidade ajustada pelo próprio atleta são adotados padrôes de locomoção frequência e amplitude de passada - com combinações correspondentes aos menores valores de $\mathrm{VO}_{2}$. Diante dessa afirmação, especula-se que o aumento da frequência de passada da corrida prolongada possa, também, estar relacionado ao desenho experimental deste estudo. A impossibilidade de o indivíduo realizar ajustes na velocidade de corrida (que neste caso foi pré-determinada), pode ter acarretado em
${ }^{*} p<0,05$ comparado à corrida prolongada. Os dados foram normalizados pelo valor máximo atingido dentro das 10 passadas do $1^{\circ} \mathrm{km}$ da corrida prolongada. 
um maior desgaste, o que pode estar associado a maior necessidade de passadas por minuto.

Não houve aumento dos sinais EMG analisados, exceto para o músculo bíceps femoral que foi maior na corrida prolongada do que na corrida do "triathlon". De acordo com KYRÖLÄINEN et al. ${ }^{11}$, o bíceps femoral tende a apresentar sua principal contribuição na corrida na potente extensão do quadril, o que the confere grande importância na fase de propulsão do movimento. Além disso, esse músculo atua excentricamente durante a fase aérea para armazenar energia elástica de forma a favorecer a fase propulsora subsequente. Assim, os ísquios-tibiais são potencialmente ativados para aumentar a força propulsiva gerando maior força resultante no sentido horizontal ${ }^{14}$.

VUORIMAA et al. ${ }^{29}$ verificaram que após corridas intensas foram utilizadas diferentes estratégias de coordenação entre os músculos agonistas e antagonistas extensores do joelho. Entretanto, diferentemente dos resultados encontrados no presente estudo, o processo de fadiga induzido por exercícios intensos levou a redução do sinal EMG dos músculos vasto lateral e vasto medial, enquanto que não foram encontradas diferenças significativas no sinal EMG do músculo bíceps femoral.

Respostas diferenciadas na ativação dos músculos flexores e extensores do joelho têm sido relatadas também em protocolos incrementais de corrida. A análise da corrida em uma ampla variedade de velocidades $\left(2.25,2.5,3.0,3.5,4.0\right.$ e $\left.4.5 \mathrm{~m} . \mathrm{s}^{-1}\right)$ não mostrou variações na amplitude do sinal EMG do músculo vasto medial em função do incremento de velocidade, enquanto que a atividade do músculo bíceps femoral aumentou com o incremento de velocidade de corrida até $3,0 \mathrm{~m} \cdot \mathrm{s}^{-1} \mathrm{e}$, após, houve uma redução da amplitude até $4,5 \mathrm{~m} \cdot \mathrm{s}^{-130}$.

Durante a execução de exercícios prolongados pode ocorrer um aumento progressivo da amplitude do sinal eletromiográfico ${ }^{31}$, o qual pode ser explicado pelo recrutamento de novas unidades motoras, substituindo a atividade das unidades motoras já fadigadas ${ }^{32}$. A fadiga representa um indicador de diminuição da força e potência, que pode ocorrer durante a execução de exercícios prolongados ${ }^{8}$.

Quando analisadas três contraçôes isométricas do músculo vasto lateral em intervalos de diferentes corridas (corrida prolongada, corrida do "triathlon" e corrida isolada), um estudo encontrou menores valores da frequência mediana e maiores valores RMS na corrida prolongada ${ }^{33}$. Uma possível explicação dos resultados reside no fato de que a corrida prolongada apresenta contraçôes concêntricas e excêntricas (diferindo, por exemplo, do ciclismo que corresponde a uma atividade predominantemente concêntrica). Isso resulta em maiores alteraçôes nas propriedades contráteis dos músculos requisitados durante a corrida.

Assim, em exercícios que predominam contrações excêntricas, pode ser observado que a função da fibra muscular acaba sendo prejudicada. Inclusive, existem vários estudos que comprovam que o principal efeito agudo do exercício excêntrico é a lesão das fibras musculares ${ }^{1,33-36}$. Esse efeito lesivo ocorre em decorrência de um aumento da sobrecarga muscular, e isso pode acarretar em um aumento na ativação das unidades motoras (aumento no recrutamento das unidades motoras e/ou na frequência de ativação neural) a fim de que novas unidades motoras assumam o papel daquelas já fatigadas e/ou lesadas. Além disso, a corrida prolongada pode induzir a altas taxas de alongamento repetitivo ocasionando lesōes ultra-estruturais as quais podem ser associadas com uma redução da habilidade do tecido muscular para realizar a contração ${ }^{33}$.

Estudos têm demonstrado que o músculo bíceps femoral apresenta-se mais sensível as alteraçōes decorrentes de mudanças na intensidade de exercício ou atividades prolongadas $s^{30,34}$. Por meio de um estudo que analisou a média do sinal EMG no início e no final de uma corrida de $5 \mathrm{~km}$, foi verificada redução significativa do sinal EMG para o músculo bíceps femoral acompanhada com a redução da velocidade de corrida, adotada como estratégia de teste, enquanto que os músculos reto femoral e vasto lateral não apresentaram diferença significativa ${ }^{34}$.

A corrida prolongada acarreta em um aumento da frequência de passada e do sinal EMG do músculo bíceps femoral quando comparada com a corrida do "triathlon". Especula-se que esses resultados estão relacionados à maior sobrecarga mecânica da corrida prolongada comparada à corrida do "triathlon", que pode ter sido responsável por uma fadiga mais elevada na corrida prolongada.

\section{Nota}

Financiamento do CNPq. 


\begin{abstract}
Comparison of electromyographic and kinematics variables between a triathlon running and a prolonged running

Running and cycling executed with the same duration and intensity may produce different biomechanical and metabolical responses during a fatigue protocol due to the mechanical overload and technical specificity of each modality. The purpose of this study was to evaluate the fatigue effects resultant from a $10 \mathrm{~km}$ running, preceded by cycling or running, on the stride pattern and electromyographic (EMG) signal. Nine male triathletes with over two years of experience participated in the study. The tests were carried out in two phases: triathlon running ( $40 \mathrm{~km}$ of cycling followed by $10 \mathrm{~km}$ of running) and prolonged running (running with equal duration of time of the $40 \mathrm{~km}$ of cycling, followed by more 10 $\mathrm{km}$ of running). Kinematic analysis (stride frequency and amplitude) and EMG analysis were performed on ten strides recorded at the 5 th $\mathrm{km}$ and at the 10 th $\mathrm{km}$ (final running period). The EMG curves were rectified and filtered prior to the calculation of the RMS curves. From the average of the ten EMG curves, the peak RMS values for the following muscles were obtained: rectus femoris, vastus lateralis, biceps femoris. Higher stride frequency and EMG signals were obtained for biceps femoris muscle on the prolonged compared to the triathlon running. These results seem related to a higher fatigue in the prolonged running due to it's higher mechanical demands.
\end{abstract}

KEY WORDS: Running; Triathlon; Fatigue; Electromyography; Kinematics.

\title{
Referências
}

1. Bentley DJ, Millet GP, Vleck VE, Mcnaughton LR. Specific aspects of contemporary triathlon. Sports Med. 2002;32:345-59.

2. Hue O, Le Gallais D, Chollet D, Boussana A, Préfaut C. The influence of prior cycling on biomechanical and cardiorespiratory response profiles during running in triathletes. Eur J Appl Physiol. 1998:77:98-105.

3. Herzog W, Guimarães ACS, Anton MG, Carter-Erdman KA. Moment-length relations of rectus femoris muscles of speed skaters/cyclists and runners. Med Sci Sports Exerc. 1991;23:1289-96.

4. Bonacci J, Green D, Saunders PU, et al. Change in running kinematics after cycling are related to alterations in running economy in triathletes. J Sci Med Sport. 2010;13:460-4.

5. Hausswirth C, Bigard AX, Berthelot M, Thomaidis C, Guezennec CY. Variability in energy cost of running at the end of a triathlon and a marathon. Int J Sports Med. 1996;17:572-9.

6. Rowlands DS, Domney B. Physiology of triathlon. In: Garrett WE, Kirdendall DT. Exercise and sport science. Philadelphia: Lippincott and Wilkins; 2000.

7. Numella AT, Paavolainen LM, Sharwood KA, Lambert MI, Noakes TD, Rusko HK. Neuromuscular factors determining $5 \mathrm{~km}$ running performance and running economy in well-trained athletes. Eur J Appl Physiol. 2006;97:1-8.

8. Rahnama N, Lees A, Reilly T. Electromyography of selected lower-limb muscle fatigue by exercise at the intensity of soccer match-play. J Electromyogr Kinesiol. 2006;16:257-63.

9. Silva SRD, Fraga CHW, Gonçalves M. Efeito da fadiga muscular na biomecânica da corrida: uma revisão. Motriz. 2005;13:225-35.

10. Smoliga JM, Myers JB, Redfern MS, Lephart SM, Reliability and precision of EMG in leg, torso, and arm muscles during running. J Electromyogr Kinesiol. 2010;20:e1-e9.

11. Kyröläinen H, Avela J, Komi PV. Changes in muscle with increasing running speed. J Sports Sci. 2005;23:1101-9.

12. Fraga CHW, Bloedow LS, Guimarães ACS, Vaz MA. Análise de variáveis cinemáticas na corrida do triathlon obtidas em prova simulada. Rev Bras Biomec. 2007;8:16-20.

13. Faulkner J, Parfitt G, Eston R. The rating of perceived exertion during competitive running scales with time. Psychophysiology. 2008;45:977-85.

14. Nicola TL, Jewison DJ. The anatomy and biomechanics of running. Clin Sports Med. 2012;31:187-201.

15. Fraga Chw, Bloedow L, Carpes F, et al. Proposta metodológica para análise cinemática e fisiológica da corrida no triathlon. Motriz. 2006;12:159-64.

Rev Bras Educ Fís Esporte, (São Paulo) 2013 Abr-Jun; 27(2):179-86 • 185 
16. Hermens HJ, Freriks B, Disselhorst-Klug C, Rau G. The SENIAM project: surface electromyography for non-invasive assessment of muscle. Proceedings of the 14th Congress of the International Society of Electrophysiology and Kinesiology; 2002; Vienna, Austria. Vienna: ISEK; 2002. [CD-ROM].

17. Merletti R. Standards for reporting EMG data. J Electromyogr Kinesiol. 1999;7:1-2.

18. Soderberg GL, Knutson LM. A guide for use and interpretation of kinesiologic electromyographic data. Phys Ther. 2000;80:485-98.

19. Neptune RR, Kautz SA, Hull ML. The effect of pedaling rate on coordination in cycling. J Biomech. 1997;30:1051-8.

20. Fraga CHW, Candotti CT, Guimarães ACS. Estudo comparativo sobre diferentes métodos de normalização do sinal eletromiográfico aplicados ao ciclismo. Rev Bras Biomec. 2008;9:?.

21. Robertson DGE, Caldwell J, Hamill G, Kamen SN. Research methods in biomechanics. Whittlesey: Human Kinetics; 2004 . v. 1.

22. Martin PE, Sanderson, DJ. Biomechanics of walking and running. In: Garrett WE, Kirdendall DT. Exercise and sport science. Philadelphia: Lippincot Willians \& Wilkins; 2000.

23. Lepers R, Theurela J, Hausswirth C, Bernardc T, Neuromuscular fatigue following constant versus variable-intensity endurance cycling in triathletes. J Sci Med Sport. 2008;11:381-9.

24. Tew GA. The effect of cycling cadence on subsequent $10 \mathrm{~km}$ running performance in well-trained triathletes. J Sports Sci Med. 2005;4:342-53.

25. Karamanidis K, Arampatzis A, Bruggemann GP. Reproducibility of electromyography and ground reaction force during various running techniques. Gait Posture. 2004;19:115-23.

26. Chumanov ES, Wille CM, Michalski MP, Heiderscheit BC. Changes in muscle activation patterns when running step rate is increased. Gait Posture. 2012;36:231-5.

27. Cavanagh PR, Kram R. Stride length in distance running: velocity, body dimensions, and added mass effects. Med Sci Sports Exerc. 1989;21:467-79.

28. Brisswalter J, Hausswirth C, Smith D, Vercruyssen F, Vallier JM. Energetically optimal cadence vs. freely-chosen cadence during cycling: effect of exercise duration. Int J Sports Med. 2000;21:60-4.

29. Vuorimaa T, Virlander R., Kurkilahti P, Vasankari T, Hâkinen K. Acute changes in muscle activation and leg extension performance after different running exercises in elite long distance runners. Eur J Appl Physiol. 2006;96:282-91.

30. Gazendam MGJ, Hof AL. Averaged EMG profiles in jogging and running at different speeds. Gait Posture. 2007;25:604-4.

31. Ravier P, Buttelli O, Jennane R, Couratier P. An EMG fractal indicator having different sensitivities to changes in force and muscle fatigue during voluntary static muscle contractions. J Electromyogr Kinesiol. 2005;15:210-21.

32. Wakeling JM; Pascual SA; Nigg M; Tscharner V. Surface EMG shows distinct populations of muscle activity when measured during sustained sub-maximal exercise. Eur J Appl Physiol. 2001;86:40-7.

33. Hausswirth C, Bigard AX, Guezennec CY. Evolution of electromyographic signal, running economy and perceived exertion during different prolonged exercises. Int J Sports Med. 2000;21:429-36.

34. Nummela AT, Heath KA, Paavolainen LM, et al. Fatigue during a 5-km running time trial. Int J Sports Med. 2008;28:738-45.

34. Vikne H, Refsnes PE, Ekmark M, Medbo JI, Gundersen V, Gundersen K. Muscular performance after concentric and eccentric exercise in trained men. Med Sci Sports Exerc. 2006;38:1770-81.

35. White KE. High hamstring tendinopathy in 3 female long distance runners. J Chiropr Med. 2011;10:93-9.

36. Lewis PB, Ruby D, Bush-Joseph CA. Muscle soreness and delayed-onset muscle soreness. Clin Sports Med. 2012;31:255-62.

ENDEREço
Carina Helena Wasem Fraga
Laboratório de Biomecânica
Escola de Educação Física e Esporte - USP
Av. Prof. Melo Moraes, 65
05508-030 - São Paulo - SP - BRASIL
e-mail: wcarina_helena@hotmail.com

Recebido para publicação: 31/07/2012

Aceito: 29/11/2012 